\title{
Proton therapy with a fixed beamline for skull-base chordomas and chondrosarcomas: outcomes and toxicity
}

\author{
Konstantin Gordon ${ }^{1,2^{*}} \mathbb{D}$, Igor Gulidov', Sergey Koryakin³ ${ }^{3}$ Daniil Smyk', Tatyana Makeenkova', Danil Gogolin , \\ Olga Lepilina ${ }^{3}$, Olga Golovanova ${ }^{3}$, Alexey Semenov' ${ }^{1}$ Sergey Dujenko ${ }^{3}$, Kira Medvedeva ${ }^{1}$ and Yuri Mardynsky ${ }^{1}$
}

\begin{abstract}
Aim: This study presents an analysis (efficacy and toxicity) of outcomes in patients with skull-base chordomas or chondrosarcomas treated with a fixed horizontal pencil proton beam.

Background: Chordomas (CAs) and chondrosarcomas (CSAs) are rare tumours that are usually located near the base of the skull and very close to the brain's most critical structures. Proton therapy (PT) is often considered the best radiation treatment for these diseases, but it is still a limited resource. Active scanning PT delivered via a fixed pencil beamline might be a promising option.

Methods: This is a single-centre experience describing the results of proton therapy for 31 patients with $C A(n=23)$ or CSA $(n=8)$ located near the base of the skull. Proton therapy was utilized by a fixed pencil beamline with a chair to position the patient between May 2016 and November 2020. Ten patients underwent resection (32.2\%), 15 patients (48.4\%) underwent R2 resection, and 6 patients had unresectable tumours (19.4\%). In 4 cases, the tumours had been previously irradiated. The median PT dose was 70 GyRBE (relative biological efficacy, 1.1) [range, 60 to 74] with 2.0 GyRBE per fraction. The mean GTV volume was $25.6 \mathrm{~cm}^{3}$ [range, 4.2-115.6]. Patient demographics, pathology, treatment parameters, and toxicity were collected and analysed. Radiation-induced reactions were assessed according to the Common Terminology Criteria for Adverse Events (CTCAE) v 4.0.

Results: The median follow-up time was 21 months [range, 4 to 52]. The median overall survival (OS) was 40 months. The 1- and 2-year OS was 100\%, and the 3-year OS was 66.3\%. Four patients died due to non-cancer-related reasons, 1 patient died due to tumour progression, and 1 patient died due to treatment-related injuries. The 1-year local control (LC) rate was 100\%, the 2-year LC rate was 93.7\%, and the 3-year LC rate was $85.3 \%$. Two patients with CSA exhibited progression in the neck lymph nodes and lungs. All patients tolerated PT well without any treatment interruptions. We observed 2 cases of $\geq$ grade 3 toxicity, with 1 case of grade 3 myelitis and 1 case of grade 5 brainstem injury.

Conclusion: Treatment with a fixed proton beam shows promising disease control and an acceptable toxicity rate, even the difficult-to-treat subpopulation of patients with skull-base chordomas or chondrosarcomas requiring dose escalation.
\end{abstract}

Keywords: Proton therapy, Fixed beam, Chordoma, Chondrosarcoma, Pencil beam, IMPT

${ }^{*}$ Correspondence: dr gordon@yahoo.com

1 Department of Proton Therapy, A. Tsyb Medical Radiological Research Centre (MRRC), 4 Korolev Street, Obninsk, Russia 249036

Full list of author information is available at the end of the article

\section{Introduction}

Chordomas and chondrosarcomas are rare among malignancies and mainly affect the skull base, sacrum bones, and vertebral column (less). These tumours original author(s) and the source, provide a link to the Creative Commons licence, and indicate if changes were made. The images or other third party material in this article are included in the article's Creative Commons licence, unless indicated otherwise in a credit line to the material. If material is not included in the article's Creative Commons licence and your intended use is not permitted by statutory regulation or exceeds the permitted use, you will need to obtain permission directly from the copyright holder. To view a copy of this licence, visit http://creativecommons.org/licenses/by/4.0/. The Creative Commons Public Domain Dedication waiver (http://creativeco mmons.org/publicdomain/zero/1.0/) applies to the data made available in this article, unless otherwise stated in a credit line to the data. 
arise from either notochordal remnants or mesenchymal cells. Because of their critical location and high local recurrence rate, even though they have a low risk of metastasis, skull-base CA and CSA are challenging to treat [1]. Their management requires a multidisciplinary approach. As gross tumour resection is not achievable in the majority of cases, RT is of vital importance. Moreover, a high total dose ( $\geq 70$ Gy) is needed to achieve adequate tumour control. Photon therapy, the most widely available irradiation treatment, usually shows inferior clinical outcomes due to the inability to strike a balance between delivering an effective dose and respecting normal tissue tolerance.

Historically, these malignancies (CA and CSA) were the first targets for proton therapy (PT) [2]. In 1999, Munzenrider et al. published the largest series of CA/ CSA patients treated with PT in Massachusetts General Hospital to date, consisting of 519 cases. The 5-year results showed local relapse-free rates of $73 \%$ for CA and $98 \%$ for CSA [3]. Another remarkable article from Loma-Linda University outlined two necessary treatment volumes, high- and low-risk, which is now mandatory used for CA/CSA target contouring [4].

Nevertheless, most of the published research describes clinical experiences related to passive-scattering protons [5]; however, active scanning techniques (e.g., spot or pencil beam) allow for better conformality, literally by "painting" the target volume and sparing surrounding normal tissues. These features help to respect dose constraints better, even in tumours located at the base of the skull, which require high doses. Compared to recent intensity-modulated photon techniques, passive-scattering PT no longer confers the advantages of efficient dose distribution; in addition, it requires specific hardware and produces contaminating secondary neutrons.

Although there is a forecast to increase the number of particle facilities in Europe to 45 centres by 2023, the use of this irradiation treatment is still infrequent [6]. Despite recent technical advances, the primary obstacles to widespread implementation are PT cost and the deficit of gantry-equipped centres. Chair-based treatment with horizontal fixed beams can be used for many disease locations, with skull bases being among them, and thus warrants further attention [7].

Since 2015, we have exclusively treated patients with the fixed pencil-beam scanning (PBS) system, the first installed in Russia [8]. We represent our initial experience with $\mathrm{PT}$ for CA/CSA patients, focusing on the clinical outcomes and toxicity of the treatment. The survival rate, disease control, and PT-related adverse events were analysed.

\section{Materials and methods}

Thirty-one patients diagnosed with CA or CSA located at the base of the skull who were treated with proton therapy at A. Tsyb Medical Radiological Research Center between May 2016 and November 2020 were identified and approved for inclusion in a retrospective analysis by a local institutional review board. Informed consent was waived due to the retrospective nature of this study. All patients were 18 years old or older with histologically or radiologically confirmed primary tumour or evidence of relapse. Eight patients were treated for recurrence after prior surgical or combined treatment, with 4 patients undergoing re-irradiation. Twenty-three patients (74.2\%) had CA, and 8 patients $(25.8 \%)$ had CSA. Of the 8 patients with CSA, $62.5 \%$ had grade 2 disease, and $37.5 \%$ had grade 1 disease. Most of the patients had ECOG (Eastern Cooperative Oncology Group) status 1 [0-3]. The patient and tumour characteristics are summarized in Table 1.

\section{Proton therapy}

Proton therapy was utilized with a fixed horizontal proton beam in a seated position, supported by cone-beam computer tomography (CT) and standard mask immobilization [9]. Diagnostic pre- and postoperative magnetic resonance images (MRI) (sequences: $\mathrm{T}_{1}-1 \mathrm{~mm}$ thickness, $\mathrm{T}_{1}$ contrast $-1 \mathrm{~mm}, \mathrm{~T}_{2}-3 \mathrm{~mm}$, Flair $-3 \mathrm{~mm}$, Fat-suppressed-3 $\mathrm{mm}$ ) were coregistered for $1 \mathrm{~mm} \mathrm{CT}$ simulation scans for optimal tumour and/or tumour bed and organ at risk (OAR) delineation. Gross tumour volume (GTV) was defined as macroscopic rest tumour or tumour bed (for R1 surgical margins). The clinical target volume (CTV) consisted of the preoperative disease extent, residual tumour defined by $\mathrm{CT}$ and MRI, and $10 \mathrm{~mm}$ margin at the area at risk anatomically adapted to the natural borders. For the first planning target volume (PTV1) generation, the corresponding CTV was expanded by a 3-mm margin, and a dose of 50 GyRBE was prescribed. Consequent PTV2 was created from the GTV, and the patient received as high a dose as possible considering the tolerance of surrounding critical structures. All delineations were cross-checked by senior physicians with a Ph.D.

The median total dose in our group was 70 GyRBE, which is sufficient for adequate tumour control. In the 3 patients who received re-irradiation, we treated PTV2 only, and for 1 patient, we performed the whole scheme with PTV1/PTV2 due to the low dose (40 Gy) and interim (15 years) from prior RT. In these cases, we used 60-66 GyRBE due to the known limits of re-irradiation. Serial OARs (i.e., spinal cord, optic nerves, chiasma, and brainstem) were allowed to receive a cumulative dose 
Table 1 Patient and treatment characteristics

\begin{tabular}{|c|c|}
\hline Patient characteristics & Number (or \%) \\
\hline Total patients & 31 \\
\hline Median follow-up time in months & $21[4-52]$ \\
\hline \multicolumn{2}{|l|}{ Gender } \\
\hline Female & $20(64.5 \%)$ \\
\hline Male & $11(35.5 \%)$ \\
\hline Median age in years & $50[27-71]$ \\
\hline Median ECOG status & $1[0-3]$ \\
\hline \multicolumn{2}{|l|}{ Histology/Radiological diagnosis } \\
\hline Chordoma & $23(74.2 \%)$ \\
\hline Chondrosarcoma & $8(25.8 \%)$ \\
\hline \multicolumn{2}{|l|}{ Surgery } \\
\hline Unresectable & $6(19.4 \%)$ \\
\hline R1 & $10(32.2 \%)$ \\
\hline R2 & $15(48.4 \%)$ \\
\hline \multicolumn{2}{|l|}{ Indication for irradiation } \\
\hline Primary & $6(19.4 \%)$ \\
\hline Adjuvant & $18(58.0 \%)$ \\
\hline Progression/recurrence (including re-RT) & $7(22.6 \%) / 4(12.9 \%)$ \\
\hline \multicolumn{2}{|l|}{ Critical OAR involvement } \\
\hline Brainstem & 6 \\
\hline Visual pathway & 7 \\
\hline Spinal cord & 1 \\
\hline Cranial nerves & 8 \\
\hline Hypophysis & 10 \\
\hline \multicolumn{2}{|l|}{ Involved anatomical site } \\
\hline Clivus & 26 \\
\hline Pyramids & 3 \\
\hline Ethmoidal bone & 4 \\
\hline Orbit & 1 \\
\hline Cervical spine & 3 \\
\hline Nasal cavity & 3 \\
\hline \multicolumn{2}{|l|}{ Symptoms } \\
\hline Headache & 15 \\
\hline Visual disfunction (diplopia) & 10 \\
\hline Facial paresis & 2 \\
\hline Motion paresis & 3 \\
\hline Otalgia & 3 \\
\hline Ptosis & 2 \\
\hline
\end{tabular}

from both courses $<120-125 \%$ from their initial constraints. Delineation of the OAR and limit prescription was performed and chosen following the recommendations by Scoccianti S. et al. [10].

Treatment was delivered in a conventional regimen, with intensity-modulated proton therapy (IMPT) based on a pencil-beam fixed line, with a $360^{\circ}$ rotating chair to position the patient. Session accuracy was ensured via $3 \mathrm{D}$ cone-beam $\mathrm{CT}$ scans and portal imaging before
Table 2 Proton therapy parameters

\begin{tabular}{ll}
\hline PT dosimetry & Number (median, range) \\
\hline Median GTV volume in cm ${ }^{3}$ & $25.6[4.2-115.6]$ \\
Median D95 & $97.8[89.2-100.0]$ \\
Median proton dose (GyRBE) & $70.0[60.0-74.0]$ \\
Dose per fraction & 2 GyRBE \\
Number of fractions & $35[30-37]$ \\
Dose to OARs & \\
Brain stem (Dmax, GyRBE) & $52.85[13.6-64.8]$ \\
Chiasma (Dmax, GyRBE) & $45.9[0.4-66.9]$ \\
Optical nerve right (Dmax, GyRBE) & $43.2[0-66.5]$ \\
Optical nerve left (Dmax, GyRBE) & $44.8[2-63.5]$ \\
Spinal cord (Dmax, GyRBE) & $20.4[0-50.9]$ \\
Temporal lobe right (Mean dose, GyRBE) & $18.0[15.0-28.0]$ \\
Temporal lobe left (Mean dose, GyRBE) & $16.5[14.0-29.3]$ \\
Hypophysis (Mean dose, GyRBE) & $52.1[32-69.8]$ \\
\hline
\end{tabular}

each field. Planning and treatment (Monte Carlo-based) were performed with the help of the Prometheus PT complex (JSC Protom, Russia). We used multifield optimized PTV-based plans, usually generated with 5-6 fields. The PT dose was prescribed to the PTV with the aim of at least $95 \%$ coverage, but in the case of meeting OAR constraints, dose limits usually prioritized target coverage depending on the individual clinical situation. Table 2 depicts the PT parameters and planning results, and Figs. 1 and 2 show the representative proton (IMPT) plans.

\section{Statistical analysis}

Study endpoints were to estimate actuarial overall survival (OS), local control (LC), and toxicity rates. Survival values were calculated using the Kaplan-Meier method with the analysis made using GraphPad Prism 8. As our study group contained a limited number of cases, we decided not to categorize patients by pathology or treatment parameters since small groups' results are difficult to assess correctly.

\section{Clinical results and toxicity}

The median follow-up time from proton irradiation was 21 months [range, 4 to 52]. The median survival time was 40 months, the 1 - and 2-year OS rates were $100.0 \%$, and the 3 -year OS rate was $66.3 \%$. Six patients died by the time of analysis, in 4 cases due to non-tumour-related reasons. One patient died due to tumour progression 27 months after PT, and 1 died due to late radiationinduced brainstem toxicity despite the tolerance only being exceeded slightly [D $\max =64.8 \mathrm{GyRBE}]$. In that case, we thought PT would be a palliative treatment 


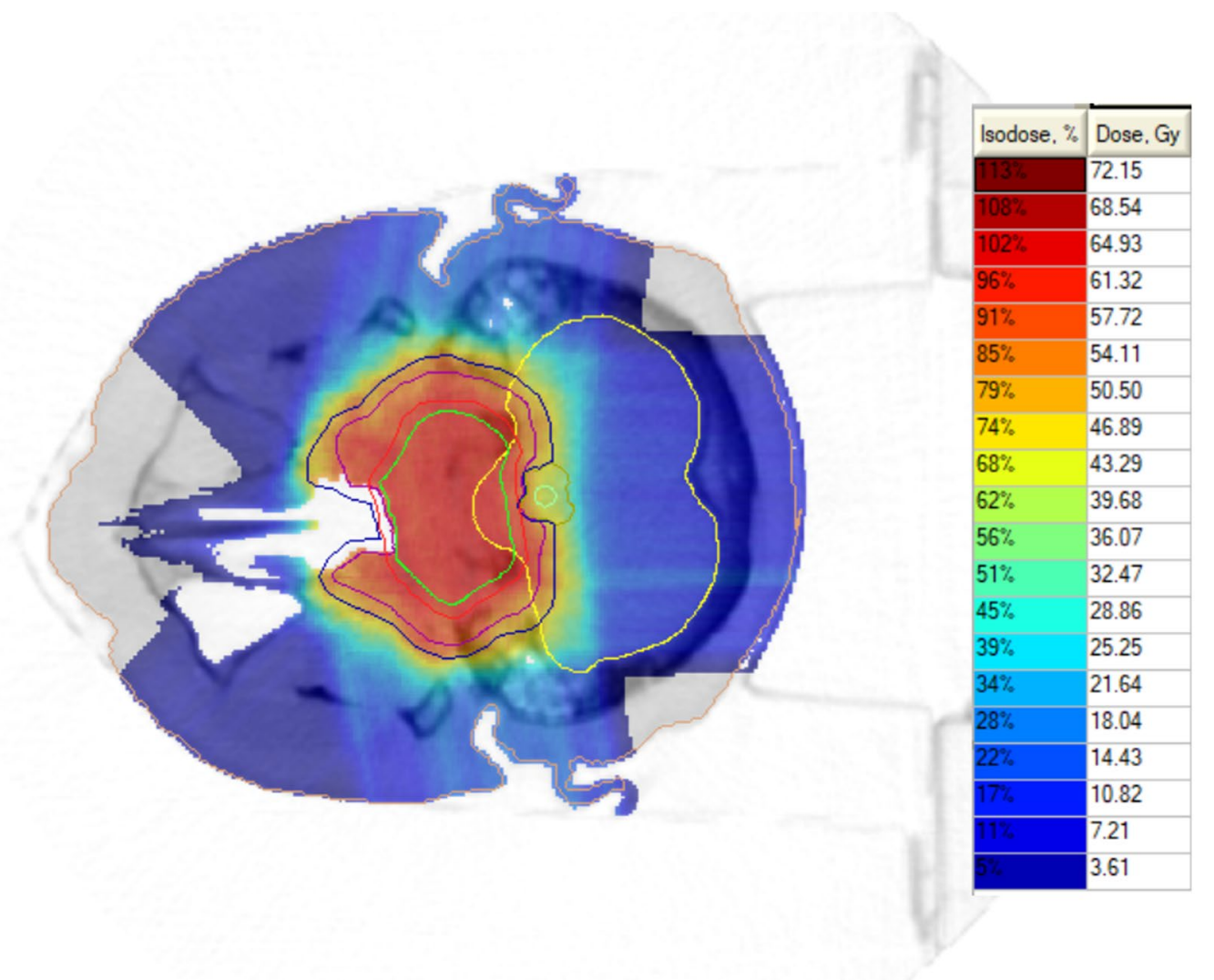

Fig. 1 Representative proton irradiation summarized plan for skull-base CA: PTV1 (dark blue line) + PTV2 (red line). Tumor volume (green contour) $=30.6 \mathrm{~cm}^{3}$ (in legends physical doses are given)

because the tumour almost entirely enveloped the brainstem, and the patient's ECOG was 3. Nevertheless, PT helped this patient survive two years, with injury occurring 22 months after PT. Despite treatment, which included bevacizumab, necrosis resulted in the patient's death at 25 months after PT.

Another 2 patients died from coronaviral pneumonia, 1 patient died due to acute gastric vessel blow-out, and 1 patient died due to heart infarct.

The 1-year LC was $100.0 \%$, the 2-year local control (LC) was $93.7 \%$, and the 3 -year LC was $85.3 \%$. Two patients with CSA also had locoregional progression in neck lymph nodes (1) and distant lung metastasis (1). They received surgery and chemotherapy and were alive without any signs of further progression at the time of the analysis.

During treatment, all patients tolerated PT well, without any course gaps. Acute toxicity was represented by grade $1(\mathrm{n}=5,16.1 \%)$ or grade $2(\mathrm{n}=1,3.2 \%)$ keratitis and laryngeal mucositis grade $2(\mathrm{n}=3,9.7 \%)$. Most events were recorded 3-6 months after finishing PT.
Late toxicity was present with grade 2 temporal lobe necrosis $(n=2,6.4 \%)$, grade 1 xerostomia $(n=1,3.2 \%)$ and grade 2 persistent headache $(\mathrm{n}=4,12.9 \%)$ developing $>12$ months after irradiation.

Additionally, we observed 2 cases of $\geq$ grade 3 late toxicity (6.4\%), namely, 1 case of grade 3 myelitis (11 months since the PT) and 1 case of grade 5 brainstem injury. The patient with grade 3 myelitis had a metal spinal fixation system, with range uncertainties due to image artefacts and density. The injury was controlled with the help of corticosteroids and bevacizumab and in 2 cases of temporal lobe necrosis. We did not observe an increase in already persistent neurological symptoms. Most patients (80.6\%) reported a subjective improvement in these symptoms after treatment. Following linear energy transfer (LET), assessment of treatment plans did not show a correlation between high-LET points and necrotic areas, excluding the patient with brainstem necrosis. 


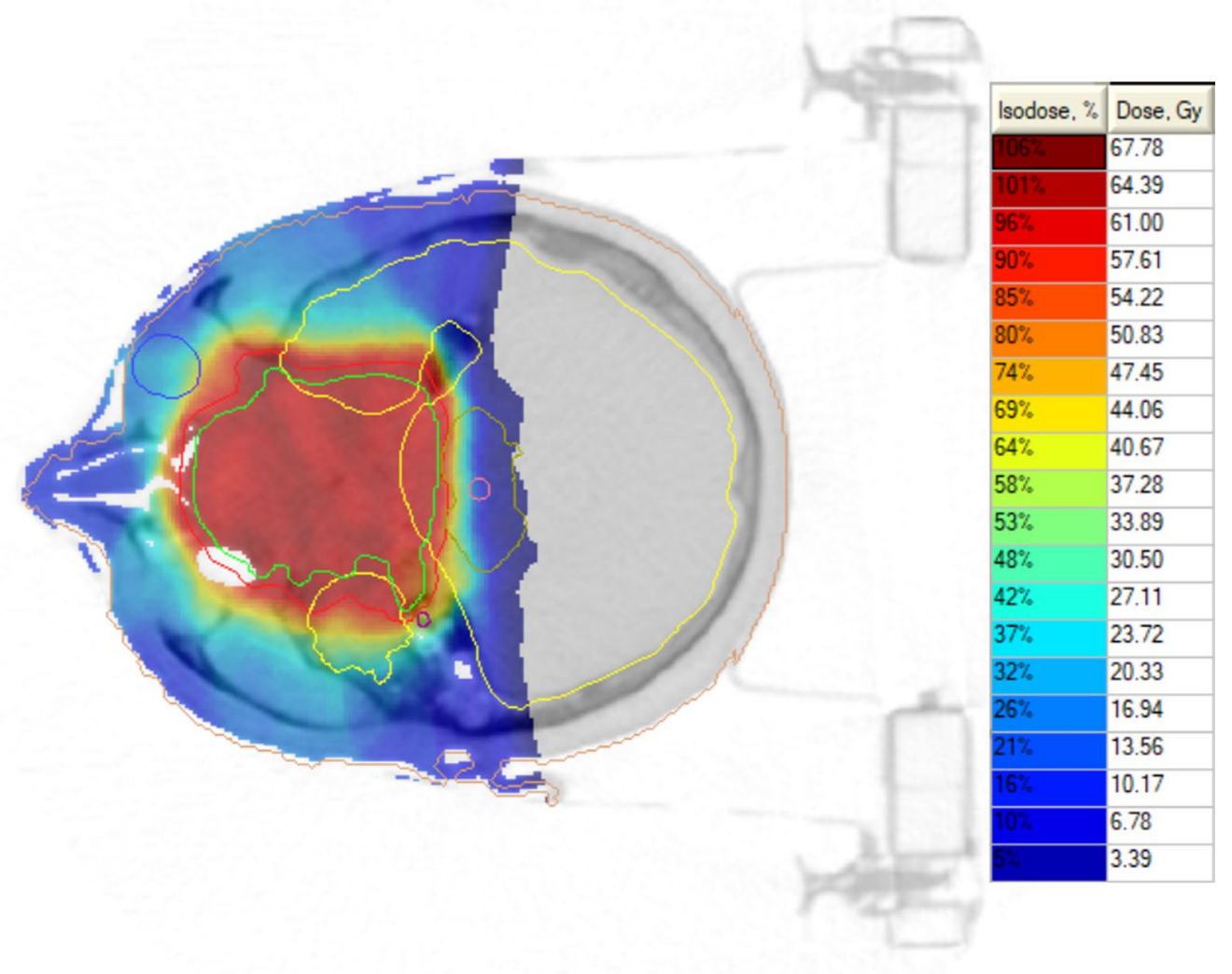

Fig. 2 Representative proton irradiation summarized plan for giant unresectable skull-base CA: PTV1 (red line). Tumor volume (green contour) $=120.8 \mathrm{~cm}^{3}$ (in legends physical doses are given)

\section{Discussion}

The primary issue of CA and CSA is local recurrence, as it determines the intensity and optimization of local therapy options such as surgery and radiotherapy. Due to known anatomical reasons, treatment of these diseases remains a complicated problem in neurooncological science, with limited surgical options available for the skullbase site. This issue has led high-dose radiation therapy to become an essential treatment with curative intent, especially after incomplete resection (or for unresectable tumours) [11].

Herein, we present our results of using PT via fixed horizontal PBS in 31 patients with skull-base CA or CSA. With a median follow-up time of 21 months, we achieved $100 \% 2$-year OS and 93.7\% 2-year LC. The three-year LC was $85.3 \%$, and the three-year OS was $66.3 \%$, with most patients $(\mathrm{n}=4)$ dying due to non-cancer-related reasons. The toxicity of PT in our cohort was moderate, and we recorded only 2 cases (6.4\%) of serious adverse events, with one treatment-related death. This was a predictable outcome due to the poor condition of the patient and deep infiltration of the brainstem before therapy. Another radiation-induced adverse reaction was grade 3 myelitis in a patient with spinal metal construction, which always causes uncertainty in PT planning [12]. Generally, most other radiation-related events were noncritical and manageable.

Conventional photon therapy is prevalent in cancer treatment due to its accessibility and cost. Current technologies such as intensity modulation or volumetric arc therapy can achieve good results with acceptable toxicity for most tumour locations. Nevertheless, in the case of tumours located at the base of the skull, external-beam therapy with conventional photons might not be able to physically achieve an adequate total dose ( $\geq 70 \mathrm{~Gy}$ ) without increasing the risk of damage to OARs. Indeed, with photon therapy, the 5-year local control or progressionfree rates are usually reported to range from 15 to $66 \%$, as the total target doses are lower than what is needed [13].

Despite the fact that Munzenrider et al. showed combined photon-proton treatment results with a passivescattering beam, the authors reported positive clinical outcomes [3]. Toxicity was not the primary study endpoint in this series; only 3 patients died due to severe 
brainstem injury, and another $8 \mathrm{had} \geq$ grade 3 toxicity. These excellent results were recently supported by other authors $[4,14-16]$.

Proton therapy can be delivered in two main ways: passive scattering (when protons are spread out by using scattering foils and conformed laterally with apertures) or, the most advanced technique, active scanning PT. This method is flexible, with a small proton beam generated and varied to treat different spots and layers in the target area. Intensity modulation became a widely used form of PT, with newly constructed centres usually equipped with such capability. However, according to the Particle Therapy Co-Operative Group statistics, nearly one-third of centres are equipped with gantry and pencil-beam systems [17]. Although it has undoubted clinical advantages, the bulk and cost of gantry systems limit the number of available PT centres [18].

Recent studies have shown that fixed beamlines can be effective from both a physical and economic point of view [19]. In 2020, Fabiano et al. presented a treatment bunker with a fixed-beam PT line and 3D-linac inside [20]. The authors revealed the optimal combination of protons and photons for head and neck cancers. However, in a lying position, a fixed beamline might limit field delivery possibilities [21]. A rotating chair can be a solution, showing promising outcomes in head and neck locations, for example [22, 23].

In 2017, Hall et al. defined tumours at the base of the skull as the targets that benefit the most from PT [24]. Treatment with an active scanning beam reduces the toxicity and can potentially increase efficacy. In 2009, Ares et al. reported outcomes of spot-scanning PT for 64 skull-base CA/CSA patients [25]. With a mean followup time of 38 months, the 5 -year LC rates were $81 \%$ for CA and $94 \%$ for CSA. Brainstem compression $(p=0.007)$ and GTV $>25 \mathrm{~cm}^{3}(p=0.03)$ were found to be risk factors for lower LC rates. The actual 5-year freedom from highgrade toxicity was $94 \%$. In our group, we had boundary tumour volume (median GTV $25.6 \mathrm{~cm}^{3}$ ), and the rate of early local control ( 1 and 2 years) was $>93 \%$. Nevertheless, over a longer period, LC decreased to $85.3 \%$ (3-year LC). Obviously, this was a result of the balance between optimal dose coverage for large tumours and the sparing of OARs.

Grosshans et al. in 2014 reported a small series of 15 patients with CA or CSA treated with spot-scanning PT [26]. Only 1 case of local recurrence and 1 case of distant failure were reported, and no cases of $>$ grade 2 toxicity were reported over a median follow-up time of 27 months.

In 2016, Feuvret et al. described a series of 159 patients with skull-base CA treated with a combination of photons and protons [15]. In 126 patients, a fixed beamline for the PT step was used, and only for 23 cases was PT delivered with the help of a gantry system. Nevertheless, the authors achieved favourable results: the 5- and 10 -year survival rates were $96.4 \%$ and $93.5 \%$, respectively, and the toxicity rate was low (a median follow-up time was 77 months). Data from the US National Cancer Data Base on CA and CSA were reported in 2019 by Palm et al. [27]. Treatment outcomes of 863 CSA patients and 715 CA cases were analysed, with 234 patients being treated with proton therapy. Proton therapy was identified as a significant factor for better outcomes in both malignancies. Unfortunately, the authors did not divide the PT group based on passive scattering and active scanning beams.

The most recent article on active scanning PT for skull-base CA and CSA was published in 2021. Parzen et al. presented their experience of IMPT with simultaneous-integrated boost delivered via gantry machine in 13 patients with small GTV volumes (median $3.4 \mathrm{~cm}^{3}$ ) [28]. With a median dose to the GTV/CTV of 72.3/50.4 GyRBE, 100\% OS and LC were recorded after a median follow-up time of 10.7 months. The absence of $>$ grade 2 toxicity events was reported, although there was 1 report of grade 2 temporal lobe necrosis.

In the recent National Comprehensive Cancer Network guidelines, CA and CSA were described as preferred targets for proton therapy [29]. Unfortunately, access to this technology, especially in developing countries, is still relatively low. Although our study cohort is limited due to the retrospective nature of this work and the inhomogeneous group of patients included, we achieved promising outcomes and report only moderate toxicity using a fixed PBS proton machine (which is typical compared to the gantry system) for a very challenging-to-treat subpopulation of patients with skull-base CA or CSA. Compared to the abovementioned articles on PT for skull-base CA and CSA, we report noninferior clinical results for our patients, the majority of whom have unfavourable tumour volumes.

\section{Conclusion}

Pencil beam proton therapy via a fixed horizontal beamline is a promising treatment for skull-base chordomas and chondrosarcomas. Our data suggest that PT is effective and safe for these malignancies, so patients with CA or CSA can be recommended for treatment at centres offering this specialized treatment without gantry systems. 


\begin{abstract}
Abbreviations
CA: Chordoma; CSA: Chondrosarcoma; CT: Computer tomography; CTCAE: Common terminology criteria for adverse events; CTV: Clinical target volume; ECOG: Eastern cooperative oncology group; Fx: Fraction; GTV: Gross tumor volume; Gy: Gray; JSC: Joint stock company; IMPT: Intensity-modulated proton therapy; LC: Local control; LET: Linear energy transfer; MRI: Magnetic resonance imaging; OAR: Organ at risk; OS: Overall survival; PBS: Pencil-beam scanning; PT: Proton therapy; PTV: Planning tumor volume; RBE: Relative biological efficacy; RT: Radiotherapy; US: United States.
\end{abstract}

\section{Acknowledgements}

Not applicable

\section{Authors' contributions}

$K G, I G$ conceived and planned the case report. KG, AS, OG, DG, OL, KM, SK, $\mathrm{SD}, \mathrm{TM}, \mathrm{DS}$ carried out the treatment and planning. KG, IG contributed to the interpretation of the results. $K G, I G$ took the lead in writing the manuscript. IG, YuM made the final approval. All authors provided critical feedback and helped shape the research, analysis and manuscript. All authors read and approved the final manuscript.

\section{Funding}

There are no funding sources.

\section{Availability of data and materials}

The data used to support the findings of this study is restricted by the Ethical Committee of A.Tsyb MRRC in order to protect patients privacy. Data is available upon request from the corresponding author for researchers, who meet the criteria for access to confidential data.

\section{Declarations}

\section{Ethics approval and consent to participate}

This study was approved by the local ethical committee and the institutional review board of A. Tsyb Medical radiological research center-branch of the National medical research radiological center, and additionally approved by the Ministry of Health of Russia, including waver of informed consent due to its retrospective nature. All procedures were performed following the ethical standards of the responsible committee on human experimentation and with the Helsinki Declaration of 1964, as revised in 2013.

\section{Consent for publication}

As corresponding author, I confirm that the manuscript has been read and approved for submission by all named authors.

\section{Competing interests}

All authors know of no competing of interest associated with this publication.

\section{Author details}

${ }^{1}$ Department of Proton Therapy, A. Tsyb Medical Radiological Research Centre (MRRC), 4 Korolev Street, Obninsk, Russia 249036. ${ }^{2}$ Department of Histology, Cytology and Embryology, Medical Institution, People's Friendship University of Russia, Moscow, Russia. ${ }^{3}$ Department of Radiophysics, A. Tsyb Medical Radiological Research Center, Obninsk, Russia.

Received: 21 August 2021 Accepted: 5 December 2021

Published online: 20 December 2021

\section{References}

1. Kremenevski N, Schlaffer S-M, Coras T, Kinfe TM, Graillon T, Buchfelder $M$, et al. Skull base chordomas and chondrosarcomas. Neuroendocrinology. 2020;110:836-47. https://doi.org/10.1159/000509386.

2. Blanchard P, Gunn GB, Lin A, Foote RL, Lee NY, Frank SJ. Proton therapy for head and neck cancers. Sem Radiat Oncol. 2018;28(1):53-63. https://doi.org/10.1016/j.semradonc.2017.08.004.

3. Munzenrider JE, Liebsch NJ. Proton therapy for tumors of the skull base. Strahlenther Onkol. 1999;175(Suppl 2):57-63. https://doi.org/10.1007/ BF03038890.
4. Hug EB, Loredo LN, Slater JD, DeVries A, Grove RI, Schaefer RA, et al. Proton radiation therapy for chordomas and chondrosarcomas of the skull base. J Neurosurg. 1999;91:432-9. https://doi.org/10.3171/jns.1999.91.3. 0432

5. Koehler AM, Schneider RJ, Sisterson JM. Flattening of proton dose distributions for large-field radiotherapy. Med Phys. 1977;4:297-301. https:// doi.org/10.1118/1.594317.

6. Durante M. Proton beam therapy in Europe: more centres need more research. Br J Cancer. 2019;120(8):777-8. https://doi.org/10.1038/ s41416-018-0329-x

7. Devicienti S, Strigari L, D'Andrea M, Benassi M, Dimiccoli V, Portaluri M. Patient positioning in the proton radiotherapy era. J Exp Clin Cancer Res. 2010;29(1):47. https://doi.org/10.1186/1756-9966-29-47

8. Gulidov IA, Mardynsky YS, Balakin VE, Galkin VN, Gogolin DV, Gordon KB, et al. New opportunities for proton therapy in Russia. Vopr Onkol. 2016;62(5):570-2 ([In Russian]).

9. Balakin VE, Belikhin MA, Pryanichnikov AA, Shemyakov AE, Strelnikova NS. Clinical application of new immobilization system in seated position for proton therapy. KnE Energy. 2018;3(2):45-51. https://doi.org/10.18502/ ken.v3i2.1790.

10. Scoccianti S, Detti B, Gadda D, Greto D, Furfaro I, Meacci F, et al. Organs at risk in the brain and their dose-constraints in adults and in children: a radiation oncologist's guide for delineation in everyday practice. Radiother Oncol. 2015;114(2):230-8. https://doi.org/10.1016/j.radonc.2015.01. 016.

11. Vrionis FD. Chordomas and chondrosarcomas of the skull base and spine Neuro Oncol. 2004;6(2):166-7. https://doi.org/10.1215/\$11528517032000 65.

12. Verburg JM, Seco J. Dosimetric accuracy of proton therapy for chordoma patients with titanium implants. Med Phys. 2013;40(7): 071727. https:// doi.org/10.1118/1.4810942.

13. Fossati P, Vavassori A, Deantonio L, Ferrara E, Krengli M, Orecchia R. Review of photon and proton radiotherapy for skull base tumours. Rep Pract Oncol Radiother. 2016;21(4):336-55. https://doi.org/10.1016/j.rpor. 2016.03.007.

14. Noël G, Feuvret L, Calugaru V, Dhermain F, Mammar H, Haie-Méder C, et al. Chordomas of the base of the skull and upper cervical spine. One hundred patients irradiated by a 3D conformal technique combining photon and proton beams. Acta Oncol. 2005;44(7):700-708. https://doi. org/10.1080/02841860500326257.

15. Feuvret L, Bracci S, Calugaru V, Bolle S, Mammar H, De Marzi L, et al. Efficacy and safety of adjuvant proton therapy combined with surgery for chondrosarcoma of the skull base: a retrospective, population-based study. Int J Radiat Oncol Biol Phys. 2016;95(1):312-21. https://doi.org/10. 1016/j.jijobp.2015.12.016.

16. Deraniyagala RL, Yeung D, Mendenhall WM, Li Z, Morris CG, Mendenhall NP, et al. Proton therapy for skull base chordomas: an outcome study from the university of Florida proton therapy institute. J Neurol Surg Part B Skull Base. 2014;75:53-7. https://doi.org/10.1055/s-0033-1354579.

17. Particle Therapy Co-operative Group. https://www.ptcog.ch/index.php/ facilities-in-operation (2021). Accessed 20 August 2021

18. Doyen J, Falk AT, Floquet V, Hérault J, Hannoun-Lévi JM. Proton beams in cancer treatments: clinical outcomes and dosimetric comparisons with photon therapy. Cancer Treat Rev. 2016;43:104-12. https://doi.org/10. 1016/j.ctrv.2015.12.007.

19. Yan S, Lu HM, Flanz J, Adams J, Trofimov A, Bortfeld T. Reassessment of the necessity of the proton gantry: analysis of beam orientations from 4332 treatments at the Massachusetts General Hospital proton center over the past 10 years. Int J Radiat Oncol Biol Phys. 2016;95:224-33.

20. Fabiano S, Balermpas P, Guckenberger M, Unkelbach J. Combined protonphoton treatments - a new approach to proton therapy without a gantry. Radiother Oncol. 2020;145:81-7. https://doi.org/10.1016/j.radonc. 2019.12.013.

21. Goitein M. Trials and tribulations in charged particle radiotherapy. Radiother Oncol. 2010;95(1):23-31. https://doi.org/10.1016/j.radonc.2009.06. 012.

22. Gordon K, Gulidov I, Semenov A, Golovanova O, Koryakin S, Makeenkova $T$, et al. Proton re-irradiation of unresectable recurrent head and neck cancers. Rep Pract Oncol Radiother. 2021;26(2):203-210. https://doi.org/ 10.5603/RPOR.a2021.0029. 
23. Sun J, Kong L, Chen Z, You D, Mao J, Guan X, et al. Clinical implementation of a $6 \mathrm{D}$ treatment chair for fixed ion beam lines. Front Oncol. 2021;11:694749. https://doi.org/10.3389/fonc.2021.694749.

24. Hall DC, Trofimov AV, Winey BA, Liebsch NJ, Paganetti H. Predicting patient-specific dosimetric benefits of proton therapy for skull-base tumors using a geometric knowledge-based method. Int J Radiat Oncol Biol Phys. 2017;97(5):1087-94. https://doi.org/10.1016/j.jirobp.2017.01. 236.

25. Ares C, Hug EB, Lomax AJ, Bolsi A, Timmermann B, Rutz H, et al. Effectiveness and safety of spot scanning proton radiation therapy for chordomas and chondrosarcomas of the skull base: first long-term report. Int J Radiat Oncol Biol Phys. 2009;75(4):1111-8. https://doi.org/10.1016/j.jprobp.2008. 12.055.

26. Grosshans DR, Zhu XR, Melancon A, Allen PK, Poenisch F, Palmer M, et al. Spot scanning proton therapy for malignancies of the base of skull: treatment planning, acute toxicities, and preliminary clinical outcomes. Int J Radiat Oncol Biol Phys. 2014;90(3):540-6. https://doi.org/10.1016/j.jprobp. 2014.07.005.

27. Palm RF, Oliver DE, Yang GQ, Abuodeh Y, Naghavi AO, Johnstone PAS. The role of dose escalation and proton therapy in perioperative or definitive treatment of chondrosarcoma and chordoma: an analysis of the National Cancer Data Base. Cancer. 2019;125(4):642-51. https://doi.org/10.1002/ cncr.31958.

28. Parzen JS, Li X, Zheng W, Ding X, Kabolizadeh P. Proton therapy for skullbase chordomas and chondrosarcomas: initial results from the beaumont proton therapy center. Cureus. 2021;13(5):e15278. https://doi.org/10. 7759/cureus.15278.

29. National Comprehensive Cancer Network. Bone Cancer (Version 1.2022) https://www.nccn.org/professionals/physician_gls/pdf/bone.pdf. Accessed 20 August 2021.

\section{Publisher's Note}

Springer Nature remains neutral with regard to jurisdictional claims in pub-

lished maps and institutional affiliations.

- fast, convenient online submission

- thorough peer review by experienced researchers in your field

- rapid publication on acceptance

- support for research data, including large and complex data types

- gold Open Access which fosters wider collaboration and increased citations

- maximum visibility for your research: over $100 \mathrm{M}$ website views per year

At BMC, research is always in progress.

Learn more biomedcentral.com/submissions 